\title{
Evaluation of microfilaricidal effects in the cornea from topically applied drugs in ocular onchocerciasis: trials with levamisole and mebendazole
}

\author{
BARRIE R. JONES, ${ }^{1}$ JOHN ANDERSON, ${ }^{2}$ AND HARALD FUGLSANG ${ }^{2}$ \\ From the ${ }^{1}$ Department of Clinical Ophthalmology, Institute of Ophthalmology, Moorfields Eye Hospital, \\ London, and ${ }^{2}$ MRC/WHO Grantholders, 77 Pennard Road, Shepherd's Bush, London
}

SUMMARY Increasing concentrations of levamisole and of mebendazole were applied to 1 eye in groups of 4 patients with ocular onchocerciasis in northern Cameroon. No effects resulted from up to $3.0 \%$ mebendazole suspensions, but $3.0 \%$ levamisole solutions rapidly caused entry of microfilariae, straightening out and subsequent opacification of previously curled-up living microfilariae, the rapid formation of typical limbal globular infiltrates, and the subsequent formation of fluffy opacities around the microfilariae. These changes are typical of all other drugs so far studied that have a microfilaricidal action on $O$. volvulus - diethylcarbamazine citrate (DEC), suramin, and metrifonate. The efficacy of $3.0 \%$ levamisole approximated to that of $0.03 \%$ DEC. This is in keeping with published observations on the filaricidal activity of these 2 compounds. It is suggested that this system of drug testing should be considered for systematic use in the search for more effective and safer drugs for onchocerciasis.

The treatment of ocular onchocerciasis is still unsatisfactory (Anderson and Fuglsang, 1977), and the World Health Organisation is urging the search for new drugs and optimum utilisation of existing drugs alone or in combinations.

The clinically observable effects of diethylcarbamazine citrate (DEC) on microfilariae (MFS) of Onchocerca volvulus are well defined, whether the drug is administered systemically (Anderson $t$ t al., 1976a) or topically (Ben-Sira et al., 1970; Aviel and David, 1972; Anderson and Fuglsang, 1973). The last authors have shown that drug effect can be judged by the entry of MFS into the cornea, the straightening out of previously curled-up living MFS, the formation of typical limbal globular infiltrates, and the subsequent formation of fluffy opacities around the MFS (Anderson and Fuglsang, 1973; Anderson et al., 1976a). These effects have also been observed during systemic treatment with suramin (Anderson et al., 1976b) and with metrifonate (Fuglsang and Anderson, 1977), both of which drugs have a microfilaricidal action.

To test the value of observing effects from drugs

Address for reprints: Professor Barrie R. Jones, Department of Clinical Ophthalmology, Moorfields Eye Hospital, City Road, London EC1V 2PD applied topically to 1 eye, preparations of levamisole and mebendazole (Fig. 1) were instilled in a small number of patients with ocular onchocerciasis.

\section{Materials and methods}

The trial was carried out at Toubourou in north Cameroon. All the patients were infected with the savanna strain of $O$. volvulus. Both levamisole and mebendazole have low water solubilities, so that a range of concentrations could not be prepared in simple aqueous solution. Antifungal imidazole drugs of low water solubility have been successfully formulated for use in the eye by dissolving the compound in chloroform, diluting in arachis oil

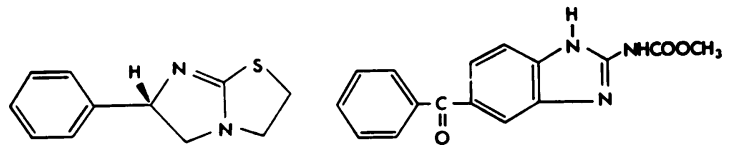

(s) $-(-)-H C L$

(a) LEMMISOLE

(D) MEBENDNZOLE

Fig. 1 Structural formula of (a) levamisole and (b) mebendazole 
$(B P)$ to the desired concentration and driving off the chloroform by heat or vacuum (Jones, 1975). This method was used for preparing levamisole drops $0 \cdot 01,0 \cdot 03,0 \cdot 1,0 \cdot 3,1 \cdot 0$, and $3.0 \%$.

Mebendazole is not sufficiently soluble in either water or arachis oil for simple formulation. It was dissolved in dimethyl sulphoxide (DMSO) and diluted in arachis oil to give suspensions of mebendazole $(0.01,0.03,0.1,0.3,1.0$, and $3.0 \%)$ each with a final concentration of $1 \%$ DMSO.

After testing to exclude immediate irritancy and toxicity in the rabbit eye by the Draize method, 8 patients were given levamisole and 4 mebendazole. The drops were instilled in the right eyes only, while the left eyes served as controls. The patients were examined with a Haag-Streit 900 slit lamp before treatment and as far as possible before each instillation. Drug effect was judged by the above-mentioned changes, which in the case of DEC therapy are usually obvious within 6 hours of the initial application of a $0.01 \%$ solution (Jones et al., 1978).

Photographs of corneal and limbal lesions were taken with a portable photographic system (Jones, Sheen, and Minassian, in preparation) using a reversed Canon FL $35 \mathrm{~mm}$ f 2.8 automatic diaphragm lens (Fig. 2) and a preset Leitz UM $10 / 0.22$ geological microscope lens (Fig. 3), in each case with an extension tube to give the magnification indicated. Diffuse oblique illumination was from a modified Vivitar 283 flash unit. Positive transparencies taken on Kodachrome 64 were enlarged on Cibachrome.

\section{Results}

\section{EXPERIMENT 1}

At midday 4 patients were given a drop of $0.01 \%$ levamisole to the right eye and $1 \%$ aureomycin ointment to the left. Six hours later there was no detectable effect, and the levamisole solution was increased to $0.3 \%$ ( 1 drop). The next morning there was still no visible effect, so the strength of levamisole was increased to $3.0 \%$, which was repeated at midday without re-examination. At $1500 \mathrm{~h}$ the previously described drug effects were obvious, and in particular 10 to 30 new discrete globular limbal infiltrates were present in the right eyes of each patient (Fig. 2). At the cessation of therapy 3 days later, most of these globular infiltrates had largely diffused away, but in 1 patient, a boy of 14,1 still well-defined infiltrate showed a haemorrhage within it (Fig. 3). Photomicrographs with magnification $\times 64$ revealed that erythrocytes had broken up the creamy, rather solid-looking exudate within the globule. Straightening of corneal MFS and subsequent development of fluffy opacities were seen in the right eyes of all these patients, but no changes were observed in the left control eyes.

EXPERIMENT 2

In order to compare the effects of levamisole with those of DEC 4 other patients were given $3 \%$ levamisole to the right eye and $1 \% \mathrm{DEC}$ to the left eye. The drops were instilled at midday and again in the afternoon on the first day. When the patients were examined the following morning drug effect was obvious in both eyes of all 4 patients. However, the DEC effects were considerably more pronounced than those of levamisole, inasmuch as there were 10 to 20 times as many new globular infiltrates in the left eyes, which also showed far more inflammatory response at the limbus on the second and third days of therapy.

\section{EXPERIMENT 3}

Mebendazole was given to 4 other patients at the same dilutions and at the same time intervals as described for levamisole in experiment $1 ; 1 \%$ aureomycin ointment was given to the left control eyes. There were no visible drug effects even after 3 days of treatment by the $3 \%$ suspension. Subsequent administration of $1 \%$ DEC drops to both eyes of 2 of these patients was rapidly followed by corneal changes typical of microfilaricidal effect.

\section{Discussion}

Levamisole (Fig. $1 a$ ) is a wide-spectrum anthelminthic given at doses ranging from $2 \cdot 5$ to $5.0 \mathrm{mg} / \mathrm{kg}$ daily for 1 to 3 days (Davis, 1973). It has also been shown to have immunopotentiating activity (Janssen, 1976) and antifilarial activity against both MFS and adult Dirofilaria immitis in dogs (Tulloch and Anderson, 1971); and also against MFS and at higher dose levels against adult Breinlia sergenti in the slow loris (Zaman and Natarajan, 1973). Lämmler et al. (1971; see also World Health Organisation, 1974) found that the minimum effective dose against the microfilariae of Litomosoides carinii infection in the multimammate rat, Mastomys natalensis, was $25 \mathrm{mg} / \mathrm{kg}$ for levamisole and 60 to $125 \mathrm{mg} / \mathrm{kg}$ for DEC, each given 5 times daily by mouth. The respective maximum tolerated doses were $160 \mathrm{mg} / \mathrm{kg} \times 5$ for levamisole and $2000 \mathrm{mg} / \mathrm{kg} \times 5$ for DEC. Levamisole also had activity against the adult worms at high dose levels, whereas DEC had none. Maertens and Wéry (1975) found that $100 \mathrm{mg}$ mebendazole given orally twice daily for 14 days had no effect on Dipetalonema perstans in a series of approximately 10 patients, whereas this dosage with $50 \mathrm{mg}$ levamisole daily in addition had a significant microfilaricidal effect. 


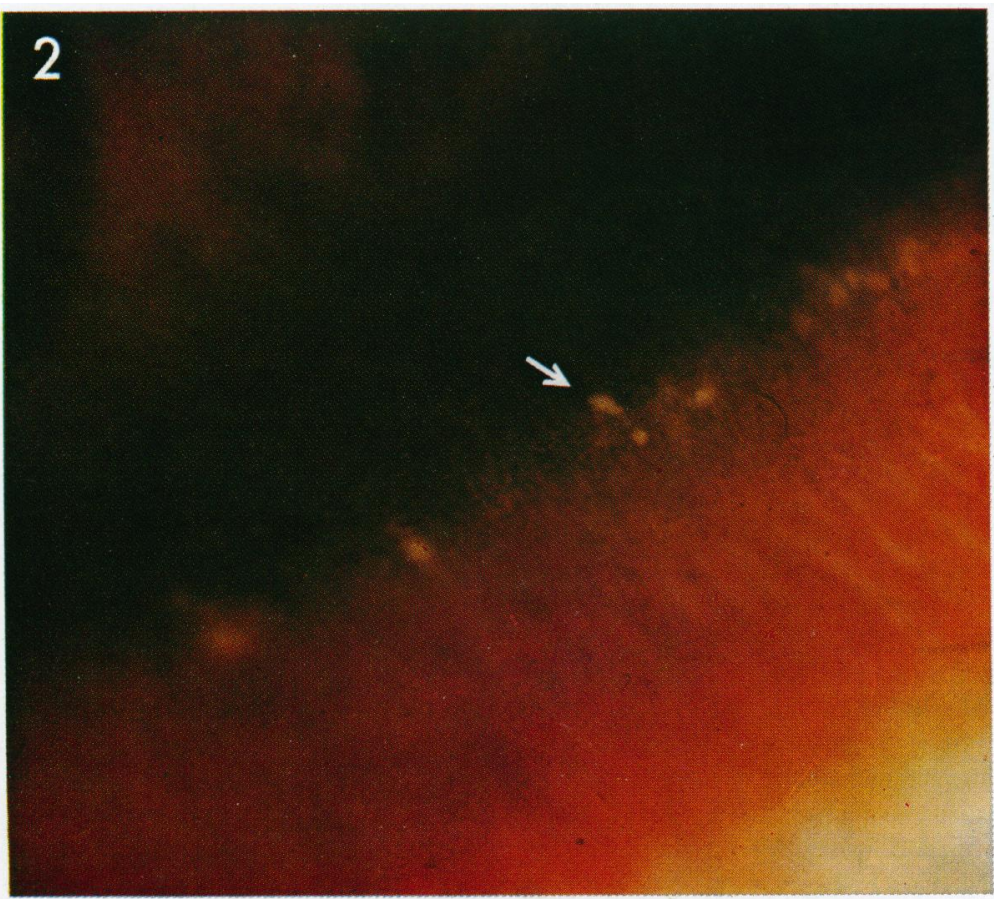

Fig. 2 Peripheral corneal and limbal globular infiltrates after 7 hours of topical treatment with levamisole $(3 \%)(\times 18.8$ : primary magnification $\times 4.7$, enlarged $\times 4$ )

Fig. 3 Limbal globular infiltrate after 3 days of treatment with topical levamisole $(3 \%)$. Haemorrhage into the globular infiltrate has broken up the rather solid exudate within the globule $(\times 64$ : primary magnification $\times 16$, enlarged $\times 4$ )

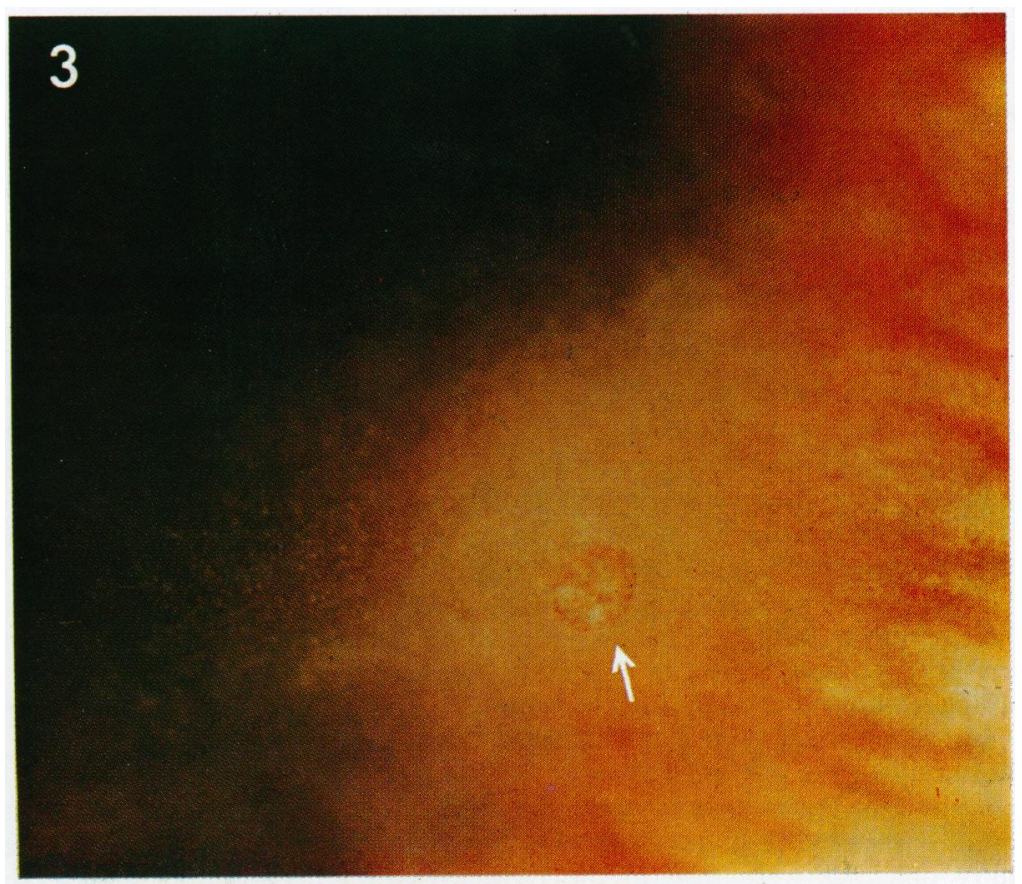


However, the same combined dosage with both levamisole and mebendazole had no demonstrable effect on microfilaria counts in 9 patients with onchocerciasis; nor did $400 \mathrm{mg}$ mebendazole combined with $300 \mathrm{mg}$ levamisole daily for 6 weeks have any effect on microfilaria counts, nor any side effects in a further 5 patients. Duke (1974a) treated a chimpanzee infected with $O$. volvulus with levamisole at a dose of $10 \mathrm{mg} / \mathrm{kg}$ intramuscularly daily for 15 days, and showed that it was both micro- and macrofilaricidal at this high dosage, which was too toxic to be considered suitable for man. The maximum dose tolerated by a single human volunteer was $2 \mathrm{mg} / \mathrm{kg}$ intramuscularly daily for 15 days, but at this level the drug had no action on MFS or adult worms (Duke, 1975).

The observation that 0.01 and $0.3 \%$ solutions of levamisole gave no effect when applied directly to the eye and that the $3 \%$ preparation was less effective than $1 \%$ DEC, together with other observations on the effect of various concentrations of DEC (Jones et al., 1978), suggest that the topical efficacy of $3 \%$ levamisole approximates to that of 0.03 to $0.1 \%$ DEC. This ratio of therapeutic activity is in keeping with the observations of Duke in the chimpanzee. There was strikingly close similarity in all respects between the changes in MFS and the associated inflammatory reactions induced by corresponding concentrations of either levamisole or DEC applied topically. This suggests that the levamisole effect is initiated by a direct action on the MFS comparable with that of DEC rather than by an immunomodulation of host defence mechanisms.

Depending on the pharmacokinetic behaviour of levamisole, it is possible that the adverse sequelae to killing MFS in the eye might be easier to regulate than is the case with DEC, but further investigation is necessary to elucidate the possibilities of useful therapy of ocular onchocerciasis with levamisole.

Mebendazole (Fig. 1b) is also a wide-spectrum anthelminthic given at a dose of $100 \mathrm{mg}$ twice daily for 3 days (Davis, 1973). Maertens and Wéry (1975) observed reduction in filaraemia due to Dipetalonema perstans in a group of 6 patients given mebendazole $100 \mathrm{mg}$ twice daily for 30 days in a randomised placebo-controlled coded trial, but no effect was seen in another group treated for only 14 days. There was complete and apparently permanent clearing in a further patient given 7 weeks' treatment. On the other hand Aguilar and Manzo (1973) were unable to detect any effect on either microfilarial or adult forms of $O$. volvulus in 19 patients treated with $400 \mathrm{mg}$ of mebendazole daily for 10 days. Similarly, Duke (1974b) found neither micro- nor macrofilaricidal effect when he administered meben- dazole tablets $27 \mathrm{mg} / \mathrm{kg}$ daily for 7 days to an experimentally infected chimpanzee. Duke suggested that the lack of action against $O$. volvulus was partly due to poor absorption of drug from the gut: only 5 to $10 \%$ is absorbed. However, the absorption from similar oral dosing (400 to $600 \mathrm{mg} 3$ times daily) for 21 to 30 days gave unequivocal ultrasonic echotomographic, immunological, and clinical evidence of destruction of Echinococcus granulosus cysts in hepatic hydatid disease in each of 4 patients treated. This effect was apparently mediated by damage to the germinal layer of the cysts (Bekhti et al., 1977).

The present investigation gave no evidence of effect on $O$. volvulus microfilariae in the cornea from $3 \%$ mebendazole applied topically as a suspension in arachis oil with $1 \%$ DMSO. It is possible that poor penetration into the cornea may have contributed to or been responsible for the lack of effect. Although $40 \mathrm{mg} / \mathrm{kg}$ mebendazole has been given daily for up to $2 \frac{1}{2}$ years in treatment of alveolar hydatid disease without adverse side effects (D. Thienpont, personal communication) there is no clear evidence that this drug affects the adult $O$. volvulus.

Since the microfilaricidal effects of DEC, suramin, metrifonate, and now levamisole can be easily demonstrated in the cornea, it is suggested that this model should be considered for systematic drug testing in onchocerciasis. When absence of topical irritancy and toxicity, and the solubility and absorption allow, the drug should ideally be applied topically to 1 eye, ascending cautiously up a half-log dilution series, leaving the fellow eye to act as a control. In this way the threshold of effective drug concentration can be determined and the doseresponse relation defined (Jones et al., 1978). It is also practicable to observe and measure unwanted side effects or sequelae to killing MFS and to study ways of minimising these.

The way in which the above drugs act on the MFS is still not understood, but it is a help to be able to observe the sequence of events in the cornea. The appearance of the typical globular infiltrates at the limbus which occur in microfilaria-loaded corneas after the administration of microfilaricidal drugs requires further studies with immunopathological examination. Detailed examination of patients with ocular onchocerciasis sometimes reveals the presence of occasional globular infiltrates in scanty numbers in untreated persons. It may be that they are related to the spontaneous death of microfilariae or to the immunopathological processes which are involved in the killing or absorption of the MFS. The question remains whether or not they are unique to onchocerciasis. 
Mr R. Watkins, chief pharmacist, Moorfields Eye Hospital, prepared the ophthalmic formulations of levamisole and mebendazole donated by Janssen Pharmaceuticals; Mr M. G. Falcon carried out the preliminary topical toxicity experiments in the rabbit eyes. The Department of Audio-Visual Communications kindly printed the illustrations.

We acknowledge the help of our assistants in the United Cameroon Republic, Mr P. Houlbai and $\mathrm{Mr}$ Antoine. The Medical Research Council and the World Health Organisation supported this study.

\section{References}

Anderson, J., and Fuglsang, H. (1973). Topical diethylcarbamazine in ocular onchocerciasis. Transactions of the Royal Society of Tropical Medicine and Hygiene, 67, 710-717.

Anderson, J., and Fuglsang, H. (1977). Ocular onchocerciasis. Tropical Diseases Bulletin, 74, 257-272.

Anderson, J., Fuglsang, H., and Marshall, T. F. de C. (1976a). Effects of diethylcarbamazine on ocular onchocerciasis. Tropenmedizin und Parasitologie, 27, 263-278.

Anderson, J., Fuglsang, H., and Marshall, T. F. de C. (1976b). Effects of suramin on ocular onchocerciasis. Tropenmedizin und Parasitologie, 27, 279-296.

Aguilar, F., and Manzo, A. G. (1973). Enfermedad de robles (oncocercosis): ensayos terapeuticos con mebendazole. 9th International Congress on Tropical Medicine and Malaria, 3, 120.

Aviel, E., and David, R. (1972). Topical diethylcarbamazine in ocular onchocerciasis. Israeli Journal of Medical Sciences, 8, 1166-1168.

Bekhti, A., Schaaps, J-P., Capron, M., Dessaint, J-P., Santoro, F., and Capron, A. (1977). Treatment of hepatic hydatid disease with mebendazole: preliminary results in four cases. British Medical Journal, 2, 1047-1051.

Ben-Sira, I., Aviel, E., Lazar, M., Lieberman, T. W., and Leopold, I. H. (1970). Topical hetrazan in the treatment of ocular onchocerciasis. American Journal of Ophthalmology, 70, 741-743.

Davis, A. (1973). Drug Treatment in intestinal helminthiases. World Health Organisation: Geneva.

Duke, B. O. L. (1974a). The effect of levamisole on Onchocerca volvulus. Transactions of the Royal Society of Tropical Medicine and Hygiene, 68, 71.

Duke, B. O. L. (1974b). Mebendazole-ineffective against Onchocerca volvulus. Transactions of the Royal Society of Tropical Medicine and Hygiene, 68, 172.

Duke, B. O. L. (1975). Further trial of levamisole against Onchocerca volvulus. Transactions of the Royal Society of Tropical Medicine and Hygiene, 69, 287-288.

Fuglsang, H., and Anderson, J. (1977). Effects of a single dose of metrifonate on the forest strain of Onchocerca volvulus in Cameroon. Tropenmedizin und Parasitologie, 28, 439-446.

Janssen, P. A. J. (1976). The levamisole story. Progress Research, 20, 347-383.

Jones, B. R. (1975). Principles in the management of oculomycocis. American Journal of Ophthalmology, 79, 719-751.

Jones, B. R., Anderson, J., and Fuglsang, H. (1978). Effects of various concentrations of diethylcarbamazine citrate applied as eye drops in ocular onchocerciasis, and the possibilities of improved therapy from continuous, nonpulsed delivery. British Journal of Ophthalmology, 62, 428-439.

Lämmler, G., Herzog, H., and Schutze, H. R. (1971). Chemotherapeutic studies on Litomosoides carinii infection of Mastomys natalensis. Bulletin of the World Health Organisation, 44, 757-763.

Maertens, K., and Wéry, M. (1975). Effect of mebendazole and levamisole on Onchocerca volvulus and Dipetalonema perstans. Transactions of the Royal Society of Tropical Medicine and Hygiene, 69, 359-360.

Tulloch, G. S., and Anderson, R. A. (1971). Acceptability of daily L-tetramisole by pound dogs infected with Dirofilaria immitis. Nature, 232, 644.

World Health Organisation Expert Committee on Filariasis: Third Report. Technical Report Series No. 352 (1974).

Zaman, V., and Natarajan, P. N. (1973). Antifilarial activity of levamisole on Breinlia sergenti and its comparison with diethylcarbamazine. Journal of Tropical Medicine and Hygiene, 76, 126. 